\title{
ORIGINAL
}

ARTICLES

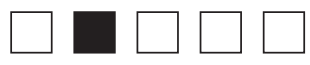

\section{HIV Care by Early-Career Family Physicians}

Kento Sonoda, MD; Zachary J. Morgan, MS; Lars E. Peterson, MD, PhD

BACKGROUND AND OBJECTIVES: Antiretroviral treatment has transformed human immunodeficiency virus (HIV) infection into a chronic disease. Prior research demonstrated a discrepancy between preparation to provide HIV care and current provision among recent residency graduates. Our study aimed to describe characteristics related to preparedness and provision of HIV care, and to identify the associations between physician and practice characteristics with current provision of HIV care among those prepared.

METHODS: We obtained data from the 2016 through 2019 American Board of Family Medicine (ABFM) National Family Medicine Graduate Survey. Our main outcome was self-reported provision of HIV care. Bivariate statistics compared differences in personal and practice characteristics with self-reported preparation for HIV care, then among those prepared, provision of HIV care. We used logistic regression to determine associations between HIV care, among those prepared, with practice and personal characteristics.

RESULTS: The response rate was $68.7 \%$ and our final sample size was 6,740 respondents. Only $25 \%$ of respondents reported preparedness in residency, and $44 \%$ of them reported current provision. Among those prepared, female gender $(\mathrm{OR}=0.604 ; 95 \% \mathrm{Cl}, 0.494-0.739)$ was associated with lower odds of practicing HIV care. Those working in high HIV prevalence areas (OR=1.718; 95\% Cl, 1.259-2.344) and in Northeast census region (OR=1.557; 95\% Cl, 1.137-2.132) had higher odds of providing HIV care.

CONCLUSIONS: Fewer than half of those prepared in residency reported currently providing HIV care. Working in a high HIV prevalence area was associated with higher odds of providing HIV care, which suggests early-career family physicians are responding to community needs.

(Fam Med. 2021;53(9):760-5.)

doi: 10.22454/FamMed.2021.415039

$\mathrm{n}$ the United States, at the end of 2018 , an estimated $1,173,900$ persons aged 13 years or more were living with human immunodeficiency virus (HIV) infection, including 161,800 who were undiagnosed. ${ }^{1}$ There were 37,968 new HIV diagnoses in 2018. ${ }^{1}$ Retention in HIV care plays a critical role in achieving good clinical outcomes and higher satisfaction with care, ${ }^{2}$

but only $57.9 \%$ of persons diagnosed with HIV received continuous HIV medical care in 2018. ${ }^{3}$ Antiretroviral treatment (ART) has transformed HIV infection into a chronic disease ${ }^{4}$ and with higher prevalence rates, its management has been moving from subspecialty care into the domain of primary care. ${ }^{5}$ In fact, patients prefer integration of HIV and primary care but HIV specialists are almost exclusively in urban areas ${ }^{6,7}$ and feel less comfortable managing common comorbid conditions, ${ }^{7,8}$ thus increasing the need to manage HIV patients in primary care. In addition, HIV quality of care, such as appropriate ART use and antimicrobial prophylaxis, is similar between generalists and HIV specialists. ${ }^{9}$

Family medicine residencies are central for increasing the number of family physicians (FPs) who will practice HIV care after graduation. ${ }^{10}$ However, a national survey of program directors (PDs) showed that teaching HIV care was a high priority for only $20 \%$, and less than $1 \%$ expected their graduates to feel prepared to provide comprehensive HIV care including ART. ${ }^{10}$ A 2016 survey of 2013 residency graduates found that nearly $30 \%$ reported being prepared to provide HIV care but less than $20 \%$ reported current provision of HIV care. ${ }^{11}$ Strengthening HIV curricula in residency training, along with closing the gap between the preparedness and the current provision of HIV care, is an urgent task.

Past work using Part D Medicare claims found that FPs prescribing ART are more likely to be
From the Department of Family Medicine, University of Pittsburgh Medical Center Shadyside, Pittsburgh, PA (Dr Sonoda); American Board of Family Medicine, Lexington, KY (Dr Peterson and Mr Morgan); and Department of Family and Community Medicine, College of Medicine, University of Kentucky, Lexington, KY (Dr Peterson). 
male, MDs, international medical graduates (IMG), practice in Northeast region and urban settings, and have been in practice for more than 10 years. $^{5} \mathrm{~A}$ fuller assessment of practice and personal characteristics associated with FPs practicing HIV care is needed to further identify barriers and enabling features. There is a substantial gap between preparation for practice and management of HIV/AIDS, ${ }^{11}$ but the impact of residency preparation on actual practice is unknown. Our study aimed to describe preparedness to provide HIV care and current provision of HIV care by early-career FPs, and to identify associations between individual and practice characteristics with current provision among those prepared.

\section{Methods}

We used data from the 2016 through 2019 American Board of Family Medicine (ABFM) National Graduate Survey. The graduate survey is administered to ABFM-certified FPs 3 years after residency graduation and is conducted to provide programmatic feedback to residency programs on the outcomes of training. ${ }^{12}$ The survey asks a broad range of questions on current practice structure, organization, location, scope of practice, and preparedness for specific areas of practice from residency training.

To build our sample, we only included FPs who primarily provided outpatient continuity care. This excluded hospitalists, those primarily in emergency rooms, or noncontinuity outpatient settings. We also excluded physicians residing outside the United States (to allow for geocoding). Standard demographics (age, gender, degree type, medical school location) were obtained from ABFM administrative data. We obtained county-level data on 2018 HIV prevalence, derived from Centers for Disease Control and Prevention data, from the AIDSVu website. ${ }^{13}$

Our main outcome was self-reported delivery of HIV care from a question asking whether the respondent personally provided, and separately was adequately prepared in residency to provide, pharmacologic management of HIV/AIDS. We used self-reported preparation for HIV care from residency training to limit the main analysis to only those who reported preparation. We categorized age as older ( $\geq 40$ years) and younger. We categorized practice size as solo, small (2-5), medium (6-20), and large $(\geq 20)$. We recoded practice organization to include low prevalence practice settings into the "other" category. We geocoded practice location, and assigned urban / rural status using the county-based Rural Urban Continuum Codes, with a code of 4 or higher being rural. We included census region to assess for prior findings of higher prescribing of ART by FPs in the Northeast. We categorized county-level HIV prevalence as low (0-149 cases per 100,000 population), medium (150-299 cases per 100,000$)$, and high (>300 cases per 100,000).

First, we compared the numbers and characteristics of those prepared and not prepared to provide HIV care. Then we used descriptive statistics to characterize our main analytic sample and then conducted bivariate analyses, using $t$ tests and $\chi^{2}$ tests, of differences by practicing HIV care among those who were prepared. We then conducted an adjusted analysis using logistic regression including all variables to assess for independent associations with providing HIV care among those who were prepared. We conducted all analyses using SAS V9.4 (SAS Corp Cary, NC). The American Academy of Family Physicians Institutional Review Board approved this study.

\section{Results}

In this study, 8,994 of 13,101 eligible FPs (68.7\% response rate) completed the graduate survey. After limiting to US-based respondents in continuity care and those with complete data, our final sample size was 6,740 .

We divided respondents into four categories: prepared and practicing, prepared but not practicing, not prepared but practicing, and not prepared and not practicing (Table 1). Of respondents, $25.0 \%(n=1,683)$ reported being adequately trained in residency to provide pharmacological management of HIV, and $16.0 \%$ $(n=1,075)$ reported providing the care in their current practice. Of those who reported the current provision, $68.7 \%(\mathrm{n}=738)$ reported being prepared in residency (Table 1); 44\% of those prepared reported current provision, whereas only $7 \%$ of those unprepared reported current provision (Table 1).

In bivariate analyses, early-career FPs who reported being prepared to provide HIV care, were more likely to be older ( $\geq 40$ years of age), male, MDs, and IMGs (Table 2). Among those who reported preparation, respondents reporting current provision of HIV care were significantly less likely to be female ( $44.1 \%$ vs $54.1 \%$; $P<.001$; Table 3$)$. Practice in high HIV prevalence areas was significantly associated with providing HIV care, as was practice in the Northeast census region. Age, degree type, location of training, practice organization, practice size, and rurality were not associated with HIV practice.

In adjusted regression analyses controlling for physician, practice, and contextual characteristics,

Table 1: Total Number of Early-Career Family Physicians Reporting Preparedness and Current Provision of HIV Care

\begin{tabular}{|l|c|c|c|c|}
\hline \multirow{2}{*}{} & \multicolumn{2}{|c|}{$\begin{array}{c}\text { Practicing HIV Care } \\
\text { n (\%) }\end{array}$} \\
\cline { 3 - 5 } & \multicolumn{2}{|c|}{ Yes } & No & Total \\
\hline \multirow{2}{*}{ Prepared for HIV Care } & Yes & $738(11.0)$ & $945(14.0)$ & $1,683(25.0)$ \\
\cline { 2 - 5 } & No & $337(5.0)$ & $4,720(70.0)$ & $5,057(75.0)$ \\
\hline & Total & $1,075(16.0)$ & $5,665(84.0)$ & 6,740 \\
\hline
\end{tabular}


Table 2: Characteristics of Early-Career Family Physicians by Self-reported Preparedness to Provide HIV Care

\begin{tabular}{|c|c|c|c|}
\hline & Prepared, $\mathbf{n}$ (\%) & $\begin{array}{c}\text { Not Prepared, } \\
\mathbf{n}(\%)\end{array}$ & $\boldsymbol{P}^{\text {Value* }}$ \\
\hline Total & 1,683 & 5,057 & \\
\hline Physician Characteristics & & & \\
\hline Age (in Years) & & & $<.001$ \\
\hline$<40$ & $1,407(83.6)$ & $4,553(90.0)$ & \\
\hline$>40$ & $276(16.4)$ & $504(10.0)$ & \\
\hline & & & $<.001$ \\
\hline Male Gender & $844(50.1)$ & $1,922(38.0)$ & \\
\hline Female Degree Type & $839(49.9)$ & $3,135(62.0)$ & \\
\hline & & & $<.001$ \\
\hline MD & $1,430(85.0)$ & $4,041(79.9)$ & \\
\hline DO & $253(15.0)$ & $1,016(20.1)$ & \\
\hline Location of Medical Training & & & $<.001$ \\
\hline United States & $1,023(60.8)$ & $3,761(74.4)$ & \\
\hline International medical graduate & $660(39.2)$ & $1,296(25.6)$ & \\
\hline
\end{tabular}

$* P$ value from $\chi^{2}$ tests

female gender remained associated with lower odds of practicing HIV care (OR=0.604; 95\% CI, 0.494-0.739; Table 4). At the practice level, only independently-owned status was significantly associated with HIV care (OR=0.664; 95\% CI, 0.457-0.963). Respondents who work in counties with the highest HIV prevalence had higher odds of providing HIV care (OR=1.718; 95\% CI, 1.2592.344). In addition, those who practice in Northeast census region had higher odds of providing HIV care (OR=1.557; 95\% CI, 1.137-2.132).

\section{Discussion}

In this large national study of recent family medicine graduates, we found that fewer than half of those who were prepared in residency to provide HIV care were doing so in practice. This discrepancy is a significant loss for both residency programs and patients. Working in a lower HIV prevalence area was associated with lower provision of HIV care despite preparedness in residency. This may indicate graduates are appropriately tailoring their practice to meet community needs.
Our study reaffirmed the past work that found FPs practicing in the Northeast region had higher odds of prescribing ART.5 In addition, our findings showed that FPs working in areas with high HIV prevalence are at higher odds of providing HIV care, however, these findings do not fit with the distribution of HIV prevalence data from the $2020 \mathrm{HIV}$ Surveillance Report. ${ }^{14}$ For instance, Northeast states were not highestprevalence states, while Georgia, Florida, and Louisiana had higher prevalence. County-level prevalence can be more important than statelevel when considering the association between the disease prevalence and provision of HIV care.

We found evidence that early-career FPs who were prepared to provide HIV care in residency were responding to community need when practicing in a high-prevalence area. This supports Barbara Starfield's idea that comprehensive primary care will look different depending on the population served. Starfield suggested that conditions in the population more frequent than 1 to 2 per 1,000 could be managed in primary care. ${ }^{15}$ Our finding that early-career
FPs in a county with a prevalence of more than 300 per 100,000 (or 3 per 1,000 ) exactly fits that definition.

Only one-fourth of early-career FPs reported adequate preparation in residency to provide pharmacological management of HIV, and even fewer early-career FPs provide HIV care in the current practice. Nearly two-thirds of those providing HIV care were prepared in residency. These findings support the significant impact of residency's curriculum on graduates' future practice. Although only $20 \%$ of PDs reported that teaching about HIV care is a high priority ${ }_{10}^{10}$ family medicine residency programs should augment HIV curriculum to meet the need of the growing HIV-infected populations, especially in high HIV prevalence areas.

There are several limitations to our work. First, this cross-sectional study design can only show associations instead of causation. Second, the term "pharmacological care of HIV/AIDS" may have been variably interpreted between early-career FPs and PDs. One-fourth of earlycareer physicians reported that their residency prepared them for pharmacological treatment of HIV/AIDS, whereas less than $1 \%$ of PDs in family medicine expected graduates to feel prepared to provide comprehensive HIV care including ART. ${ }^{10}$ In the graduate survey, respondents might have interpreted the subjective term "adequate" for the training to prepare for HIV practice. Third, recall bias may exist since respondents self-assessed their preparedness in residency 3 years after the graduation. Lastly, early-career FPs may not be representative of all FPs providing HIV care given the positive correlation between years in practice and current provision of HIV care seen in prior studies.

Only one-sixth of early-career FPs are currently providing HIV care. Working in a high HIV prevalence area was associated with higher odds of providing HIV care, which suggests early-career family physicians are responding to community needs. 
Table 3: Personal and Practice Characteristics of Early-Career Family Physicians Who Selfreported Preparedness to Provide HIV Care, by Provision of HIV Care

\begin{tabular}{|c|c|c|c|}
\hline & $\begin{array}{c}\text { Prepared and } \\
\text { Practicing, } \mathbf{n}(\%)\end{array}$ & $\begin{array}{l}\text { Prepared and Not } \\
\text { Practicing, n (\%) }\end{array}$ & $P$ Value \\
\hline Total & 738 & 945 & \\
\hline \multicolumn{4}{|l|}{ Physician Characteristics } \\
\hline Age (in Years) & & & .351 \\
\hline$<40$ & $624(84.6)$ & $783(82.9)$ & \\
\hline$>40$ & $114(15.4)$ & $162(17.1)$ & \\
\hline Gender & & & $<.001$ \\
\hline Male & $410(55.6)$ & $434(45.9)$ & \\
\hline Female & $328(44.4)$ & $511(54.1)$ & \\
\hline Degree Type & & & .219 \\
\hline MD & $636(86.2)$ & $794(84.0)$ & \\
\hline $\mathrm{DO}$ & $102(13.8)$ & $151(16.0)$ & \\
\hline Location of Medical Training & & & .388 \\
\hline United States & $440(59.6)$ & $583(61.7)$ & \\
\hline International medical graduate & $298(40.4)$ & $362(38.3)$ & \\
\hline \multicolumn{4}{|l|}{ Practice/Contextual Characteristics } \\
\hline Practice Organization & & & .074 \\
\hline $\begin{array}{l}\text { Hospital-/health system-owned medical practice (not including } \\
\text { managed care or HMO) }\end{array}$ & $279(37.8)$ & $368(38.9)$ & \\
\hline Independently-owned medical practice & $91(12.3)$ & $154(16.3)$ & \\
\hline Managed care/HMO practice & $70(9.5)$ & $88(9.3)$ & \\
\hline $\begin{array}{l}\text { Academic health center / faculty practice (residency or university } \\
\text { teaching environment) }\end{array}$ & $79(10.7)$ & $90(9.5)$ & \\
\hline Federally qualified health center or look-alike & $106(14.4)$ & $100(10.6)$ & \\
\hline Other & $113(15.3)$ & $145(15.3)$ & \\
\hline Practice Size & & & .985 \\
\hline Solo & $26(3.5)$ & $35(3.7)$ & \\
\hline $2-5$ providers & $277(37.5)$ & $350(37.0)$ & \\
\hline 6-20 providers & $258(35.0)$ & $337(35.7)$ & \\
\hline$>20$ providers & $177(24.0)$ & $223(23.6)$ & \\
\hline HIV Prevalence & & & .001 \\
\hline Low $(0-<150 / 100 \mathrm{~K})$ & $149(20.2)$ & $252(26.7)$ & \\
\hline Medium $(150-<300 / 100 \mathrm{~K})$ & $232(31.4)$ & $312(33.0)$ & \\
\hline High $(>300 / 100 \mathrm{~K})$ & $357(48.4)$ & $381(40.3)$ & \\
\hline Urban/Rural & & & .277 \\
\hline Urban & $629(85.2)$ & $787(83.3)$ & \\
\hline Rural & $109(14.8)$ & 158 (16.7) & \\
\hline Census Region & & & .008 \\
\hline Northeast & $127(17.2)$ & $115(12.2)$ & \\
\hline Midwest & $118(16.0)$ & $192(20.3)$ & \\
\hline South & $290(39.3)$ & 366 (38.7) & \\
\hline West & $203(27.5)$ & $272(28.8)$ & \\
\hline
\end{tabular}


Table 4: Adjusted Associations With Provision of HIV Care Among Early-Career Family Physicians Who Self-Reported Preparedness to Provide HIV Care

\begin{tabular}{|c|c|c|}
\hline & Odds Ratio (95\% Cl) & $P$ Value \\
\hline \multicolumn{3}{|l|}{ Physician Characteristics } \\
\hline \multicolumn{3}{|l|}{ Age (in Years) } \\
\hline$<40$ & Reference & \\
\hline$>40$ & $0.817(0.621-1.074)$ & .351 \\
\hline \multicolumn{3}{|l|}{ Gender } \\
\hline Male & Reference & \\
\hline Female & $0.604(0.494-0.739)$ & $<.001$ \\
\hline \multicolumn{3}{|l|}{ Degree Type } \\
\hline $\mathrm{MD}$ & $0.912(0.678-1.227)$ & .219 \\
\hline $\mathrm{DO}$ & Reference & \\
\hline \multicolumn{3}{|l|}{ Location of Medical Training } \\
\hline United States & $1.049(0.840-1.312)$ & .388 \\
\hline International medical graduate & Reference & \\
\hline \multicolumn{3}{|l|}{ Practice/Contextual Characteristics } \\
\hline \multicolumn{3}{|l|}{ Practice Organization } \\
\hline $\begin{array}{l}\text { Hospital/health system-owned medical practice (not including managed care or } \\
\text { HMO) }\end{array}$ & $0.970(0.718-1.309)$ & .841 \\
\hline Independently-owned medical practice & $0.664(0.457-0.963)$ & .031 \\
\hline Managed care/HMO practice & $0.965(0.631-1.474)$ & .868 \\
\hline $\begin{array}{l}\text { Academic health center/faculty practice (residency or university teaching } \\
\text { environment) }\end{array}$ & $1.063(0.702-1.611)$ & .772 \\
\hline Federally qualified health center or look-alike & $1.320(0.895-1.945)$ & .161 \\
\hline Other & Reference & \\
\hline \multicolumn{3}{|l|}{ Practice Size } \\
\hline Solo & $1.101(0.620-1.956)$ & .743 \\
\hline $2-5$ providers & $1.087(0.824-1.434)$ & .556 \\
\hline $6-20$ providers & $1.034(0.791-1.354)$ & .805 \\
\hline$>20$ providers & Reference & \\
\hline \multicolumn{3}{|l|}{ HIV Prevalence } \\
\hline Low $(0-<150 / 100 \mathrm{~K})$ & Reference & \\
\hline Medium $(150-<300 / 100 \mathrm{~K})$ & $1.263(0.929-1.717)$ & .136 \\
\hline High $(>300 / 100 K)$ & $1.718(1.259-2.344)$ & $<.001$ \\
\hline \multicolumn{3}{|l|}{ Urban/Rural } \\
\hline Urban & Reference & \\
\hline Rural & $1.135(0.824-1.562)$ & .277 \\
\hline \multicolumn{3}{|l|}{ Census Region } \\
\hline Northeast & $1.557(1.137-2.132)$ & .006 \\
\hline Midwest & $0.873(0.645-1.180)$ & .377 \\
\hline South & Reference & \\
\hline West & $1.022(0.787-1.327)$ & .871 \\
\hline
\end{tabular}


This information can help PDs tailor training for residents who know where they are going to practice to meet patient needs. Further studies to explore potential barriers to provide HIV care may be needed.

FUNDING STATEMENT: Dr Sonoda received financial support from the American Board of Family Medicine Foundation.

CORRESPONDING AUTHOR: Ad dress correspondence to Dr Kento Sonoda, 5215 Centre Avenue, Pittsburgh, PA 15232. 412-623-2287. Fax: 412-623-6629. kento.sonoda.md@gmail.com.

\section{References}

1. Centers for Disease Control and Prevention. Estimated HIV incidence and prevalence in the United States, 2014-2018. HIV Surveillance Supplemental Report 2020;25(No. 1) Published May 2020. Accessed March 9, 2021. http://www.cdc.gov/hiv/library/reports/hiv-surveillance.html.

2. Ding L, Landon BE, Wilson IB, Hirschhorn LR, Marsden PV, Cleary PD. The quality of care received by HIV patients without a primary provider. AIDS Care. 2008;20(1):35-42. doi:10.1080/09540120701439295

3. Centers for Disease Control and Prevention Monitoring selected national HIV prevention and care objectives by using HIV surveillance data-United States and 6 dependent areas, 2018. HIV Surveillance Supplemental Report 2020;25(No. 2). Published May 2020. Accessed March 9, 2021. http://www.cdc.gov/hiv/library/ reports/hiv-surveillance.html.
4. Deeks SG, Lewin SR, Havlir DV. The end of AIDS: HIV infection as a chronic disease. Lancet. 2013;382(9903):1525-1533. doi:10.1016 S0140-6736(13)61809-7

5. Egan K, Jetty A, Petterson S, Liaw W, Wingrove PM, Bazemore A. Family medicine's contribution to the HIV workforce. Fam Med. 2019;51(6):532-534. doi:10.22454/ FamMed.2019.918847

6. Walensky RP, McQuillen DP, Shahbazi S, Goodson JD. Where is the ID in COVID-19? Ann Intern Med. 2020;173(7):587-589. doi:10.7326/ M20-2684

7. Cheng QJ, Engelage EM, Grogan TR, Currier JS, Hoffman RM. Who provides primary care? an assessment of HIV patient and provider practices and preferences. J AIDS Clin Res. 2014;5(11):366. doi:10.4172/2155-6113.1000366

8. Fultz SL, Goulet JL, Weissman S, et al. Differences between infectious diseases-certified physicians and general medicine-certified physicians in the level of comfort with providing primary care to patients. Clin Infect Dis. 2005;41(5):738-743. doi:10.1086/432621

9. Kerr CA, Neeman N, Davis RB, et al. HIV quality of care assessment at an academic hospital: outcomes and lessons learned. Am J Med Qual. 2012;27(4):321-328. doi:10.1177/1062860611425714

10. Prasad R, D'Amico F, Wilson SA, et al. Training family medicine residents in HIV primary care: a national survey of program directors. Fam Med. 2014;46(7):527-531.

11. Peterson LE, Fang B, Puffer JC, Bazemore AW. Wide gap between preparation and scope of practice of early career family physicians. J Am Board Fam Med. 2018;31(2):181-182. doi:10.3122/jabfm.2018.02.170359
12. Weidner AKH, Chen FM, Peterson LE. Developing the National Family Medicine Graduate Survey. J Grad Med Educ. 2017;9(5):570-573. doi:10.4300/JGME-D-17-00007.1

13. Tools and Resources. AIDSVu. Accessed March 9, 2021. https://aidsvu.org/resources/\#/tab-data.

14. HIV Surveillance Report, 2018 (Updated), Vol. 31. Published May 2020. Accessed March 9, 2021. http://www.cdc.gov/hiv/library/reports/ hiv-surveillance.html.

15. Starfield B. Primary Care: Balancing Health Needs, Services, and Technology. Rev. ed. New York: Oxford University Press; 1998 